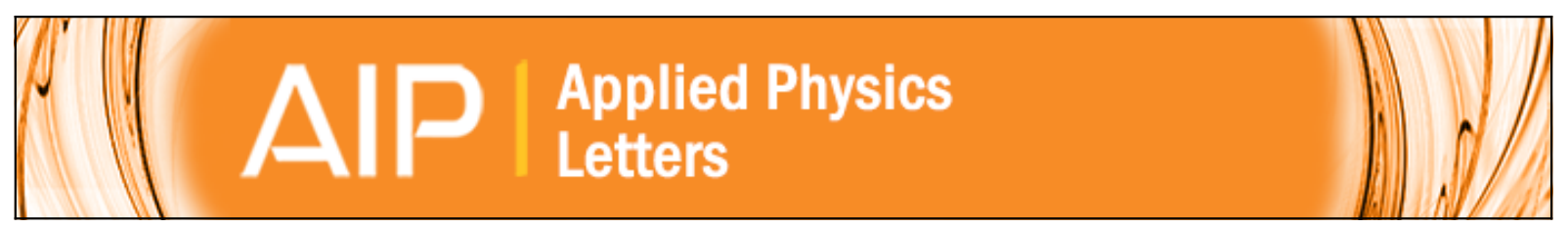

\title{
Chemical solution deposition of ferroelectric yttrium-doped hafnium oxide films on platinum electrodes
}

S. Starschich, D. Griesche, T. Schneller, R. Waser, and U. Böttger

Citation: Applied Physics Letters 104, 202903 (2014); doi: 10.1063/1.4879283

View online: http://dx.doi.org/10.1063/1.4879283

View Table of Contents: http://scitation.aip.org/content/aip/journal/apl/104/20?ver=pdfcov

Published by the AIP Publishing

\section{Articles you may be interested in}

Ferroelectricity in yttrium-doped hafnium oxide

J. Appl. Phys. 110, 114113 (2011); 10.1063/1.3667205

Ferroelectricity in hafnium oxide thin films

Appl. Phys. Lett. 99, 102903 (2011); 10.1063/1.3634052

Evaluation of the space charge trap energy levels in the ferroelectric films

J. Appl. Phys. 106, 014108 (2009); 10.1063/1.3152797

Structural, ferroelectric, dielectric, and magnetic properties of BiFeO 3 / Bi $3.15 \mathrm{Nd} 0.85 \mathrm{Ti} 3 \mathrm{O} 12$ multilayer films derived by chemical solution deposition

J. Appl. Phys. 105, 084109 (2009); 10.1063/1.3110074

Room temperature ferroelectric properties of Mn-substituted Bi Fe O 3 thin films deposited on Pt electrodes using chemical solution deposition

Appl. Phys. Lett. 88, 262908 (2006); 10.1063/1.2218819

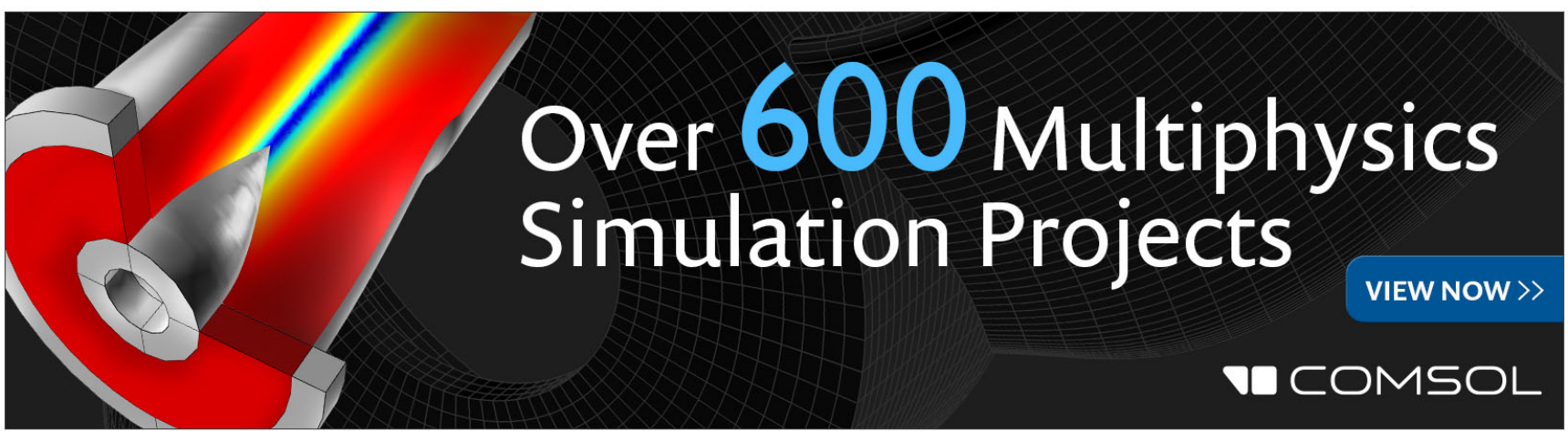




\title{
Chemical solution deposition of ferroelectric yttrium-doped hafnium oxide films on platinum electrodes
}

\author{
S. Starschich, ${ }^{1}$ D. Griesche,${ }^{1}$ T. Schneller, ${ }^{1}$ R. Waser,,${ }^{1,2}$ and U. Böttger ${ }^{1}$ \\ ${ }^{1}$ Institut für Werkstoffe der Elektrotechnik 2, RWTH Aachen University, Sommerfeldstraße 24, \\ D-52074 Aachen, Germany \\ ${ }^{2}$ Peter Grünberg Institut 7, Forschungszentrum Jülich GmbH, D-52425 Jülich, Germany
}

(Received 17 April 2014; accepted 11 May 2014; published online 21 May 2014)

Ferroelectric hafnium oxide films were fabricated by chemical solution deposition with a remnant polarization of $>13 \mu \mathrm{C} / \mathrm{cm}^{2}$. The samples were prepared with $5.2 \mathrm{~mol} \%$ yttrium-doping and the thickness varied from $18 \mathrm{~nm}$ to $70 \mathrm{~nm}$. The hafnium oxide layer was integrated into a metal-insulator-metal capacitor using platinum electrodes. Due to the processing procedure, no thickness dependence of the ferroelectric properties was observed. To confirm the ferroelectric nature of the deposited samples, polarization, capacitance, and piezoelectric displacement measurements were performed. However, no evidence of the orthorhombic phase was found which has been proposed to be the non-centrosymmetric, ferroelectric phase in $\mathrm{HfO}_{2}$. C 2014 AIP Publishing LLC . [http://dx.doi.org/10.1063/1.4879283]

Ferroelectric memories are promising candidates for future high density, low power, and nonvolatile memory technology. The perovskite structured lead zirconate titanate (PZT) is up to now the state-of-the-art material for ferroelectric memory fabrication. ${ }^{1}$ The drawback of PZT and many other ferroelectric materials is the difficult integration into the CMOS technology and the partially toxic materials used, such as lead. As a consequence, the integration density is much lower compared to, e.g., NAND Flash. ${ }^{2}$ Hafnium oxide is widely used as a dielectric in CMOS and DRAM devices and can overcome the common integration problems for ferroelectric materials due to its CMOS compatibility. ${ }^{3}$ Recently, the ferroelectric nature of doped hafnium oxide was shown for several dopants $\left(\mathrm{Si}^{4}{ }^{4} \mathrm{Y},{ }^{5} \mathrm{Zr},{ }^{6} \mathrm{Al},{ }^{7} \mathrm{Gd},{ }^{8}\right.$ and $\mathrm{Sr}^{9}$ ) deposited by atomic layer deposition (ALD) and sputtering. ${ }^{10}$

In this Letter, we report our results on $5.2 \%$ yttrium doped hafnium oxide deposited by chemical solution deposition (CSD) using platinum electrodes. CSD is an inexpensive and flexible deposition technique with good adjustability of concentration and stoichiometry and is widely used for ferroelectric and piezoelectric ceramic thin films. ${ }^{11}$ The dopant ratio was chosen, because it was reported to exhibit the highest remanent polarization in ALD deposited samples. ${ }^{5}$ To confirm the ferroelectric properties of the deposited films polarization-voltage (P-V), capacitance-voltage $(\mathrm{C}-\mathrm{V})$ and piezoelectric displacement measurements were performed.

Planar metal-insulator-metal (MIM) capacitors were manufactured on 1 in. $^{2}$ oxidized silicon substrates. $100 \mathrm{~nm}$ thick platinum bottom electrodes with a titanium dioxide adhesion layer were deposited by sputtering from a 6 in. target with $200 \mathrm{~W}$ at $150^{\circ} \mathrm{C}$. For the preparation of the precursor solution, a hybrid-type routine was applied. ${ }^{12}$ For the solgel-educt (hafnium ethoxide, Alfa Aesar, 99.9\%), all preparations were carried out under inert gas atmosphere by the use of standard Schlenk techniques and a glove box. The desired amount of hafnium ethoxide was weighted into a Schlenk flask and dry ethanol was added. After heating at $60^{\circ} \mathrm{C}$ for $30 \mathrm{~min}$ in an oil bath, a transparent solution was formed, which was stabilized by adding one equivalent of 2,4-pentanedionate (Sigma-Aldrich, GC-grade) per hafnium ion. Yttrium 2,4-pentanedionate (Sigma Aldrich, 99.95\%) was dissolved in a mixture $(5: 1)$ of propionic acid (Merck, f. s.) and propionic anhydride (Merck, f. s.) at $100^{\circ} \mathrm{C}$. The two solutions were combined and the concentration was adjusted to $0.1 \mathrm{~mol}$ with propionic acid. The resulting solution was spin coated on the substrate and heated up in a RTP

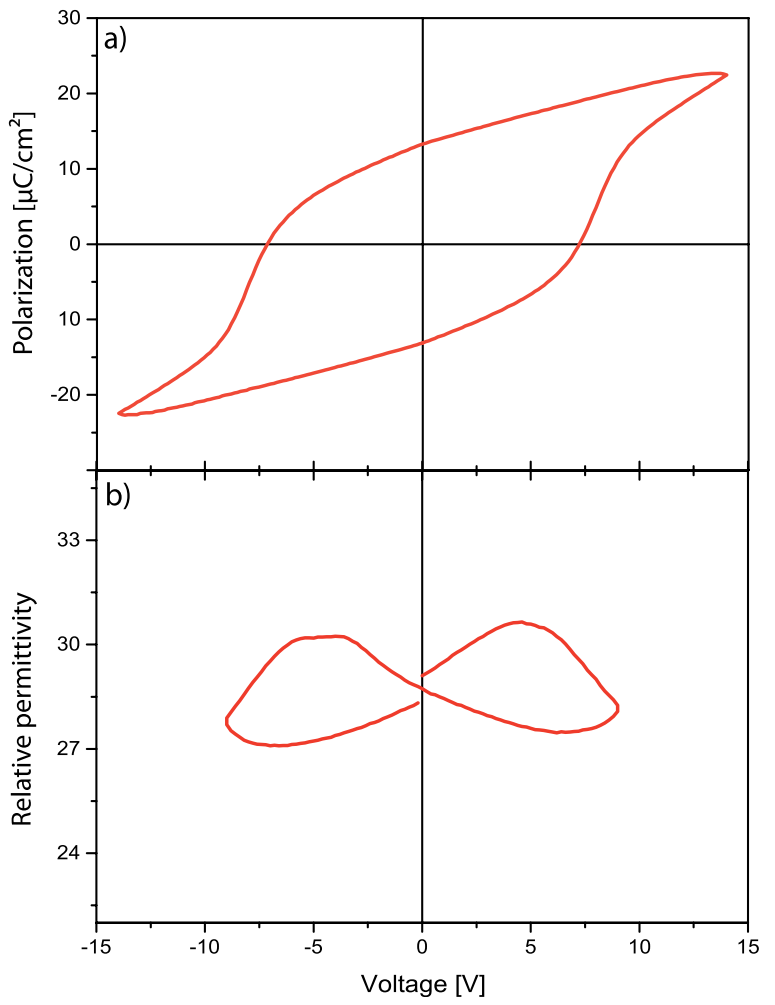

FIG. 1. Characteristic (a) polarization and (b) relative permittivity results for a $\mathrm{Pt} / \mathrm{HfO}_{2}(35 \mathrm{~nm}) / \mathrm{Pt}$ sample measured after 1000 wake up cycles. The hysteresis shows a remanent polarization of $>13 \mu \mathrm{C} / \mathrm{cm}^{2}$ and a coercive voltage of $\pm 7.5 \mathrm{~V}$. The $\varepsilon_{r}$-curve exhibits the typical double peaks during ferroelectric switching. 


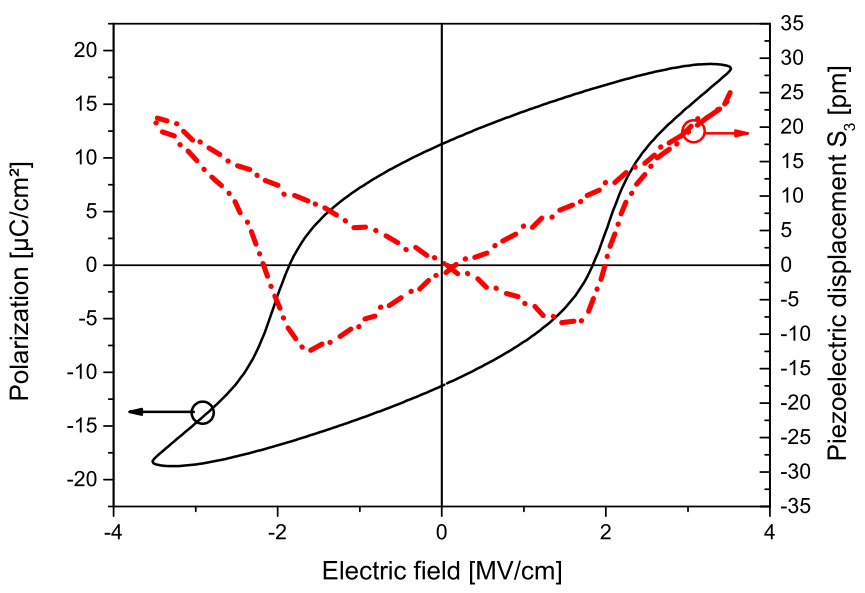

FIG. 2. Piezoelectric measurement (dashed line) with the corresponding P-V curve (straight line) for a $70 \mathrm{~nm}$ Y: $\mathrm{HfO}_{2}$ MIM capacitor clearly reveals a butterfly hysteresis for the mechanical displacement. The both minima of the displacement curve indicate the polarization switching. The linear behavior reveals the piezoelectric properties of the sample.

at $700{ }^{\circ} \mathrm{C}$ for $5 \mathrm{~min}$ in an oxygen atmosphere. Approximately, $7 \mathrm{~nm} \mathrm{Y}: \mathrm{HfO}_{2}$ was deposited by each spin coating step. The film thickness was adjusted by repeating the deposition steps. The patterned top $50 \mathrm{~nm}$ platinum electrodes were deposited by a negative lift-off process at room temperature.

The P-V and piezoelectric measurements were performed using an aixACCT Systems TF Analyzer 2000 and an aixACCT Systems aixDBLI double beam laser interferometer, ${ }^{13}$ respectively. The capacitance measurements were recorded with a HP4284A LCR meter, whereas the grazing incidence $\mathrm{X}$-ray diffractions (GI-XRD) and X-ray reflectivity (to determine the layer thickness) data were measured by PANalytical Xố̉Pert Pro. The P-V and C-V curves were measured at a frequency of $1 \mathrm{kHz}$ with a $50 \mathrm{mV}$ ac probing signal for the $\mathrm{C}-\mathrm{V}$ measurements on device areas of $2.5 \times 10^{-4} \mathrm{~cm}^{2}$.
Fig. 1 shows characteristic P-V and C-V curves for a $35 \mathrm{~nm}$ thick $\mathrm{Y}: \mathrm{HfO}_{2}$ layer with a remanent polarization of $>13 \mu \mathrm{C} / \mathrm{cm}^{2}$ (Fig. 1(a)) and the characteristic double peak for the relative permittivity $\varepsilon_{r}$, calculated from the measured capacitance (Fig. 1(b)). ${ }^{14}$ The so far reported $\mathrm{HfO}_{2}$ based ferroelectric MIM structures used $\mathrm{TiN}$ as a bottom electrode. By the use of platinum electrodes, we can exclude that oxygen scavenging, as reported for $\mathrm{TiN}_{\mathrm{x}} / \mathrm{HfO}_{2}$ interfaces, ${ }^{15}$ is the primary cause for ferroelectricity in $\mathrm{HfO}_{2}$ based films. The results of a piezoelectric displacement measurement are shown in Fig. 2, in order to confirm the ferroelectric properties of the deposited films. A butterfly loop is clearly visible with its minima at the ferroelectric switching points. Contrary to other reported displacement hysteresis, ${ }^{4,6}$ a linear behavior is observed outside the switching range unequivocally identifying the piezoelectric nature.

To achieve the maximum remanent polarization, an amount of "wake up" cycles is necessary as reported by Zhou et al. ${ }^{16}$ for Si-doped $\mathrm{HfO}_{2}$. Fig. 3 shows the influence of cycling on the hysteresis for a $56 \mathrm{~nm}$ thick Y:HfO2 sample. At an initial sweep with a maximum voltage of $16 \mathrm{~V}$, no ferroelectric hysteresis is observed (Fig. 3(a)). After 1000 cycles at the same conditions, a hysteresis occurs with clear current peaks during polarization switching (Fig. 3(b)). The remanent polarization can be further improved by more cycles at a higher voltage shown in Figs. 3(c) and 3(d). Due to the influence of the wake up cycles, an equal amount of cycles at the same electric field is performed for the samples shown in Fig. 4. For the reason of clarity, only three different $Y: \mathrm{HOO}_{2}$ thicknesses are shown in Fig. 4. Contrary to the ALD deposited samples, ${ }^{17}$ the CSD samples show no reduction of the remanent polarization with higher film thicknesses. This is possibly attributed to the high temperature treatment after each layer deposition resulting in a step by step crystallization of the $\mathrm{Y}: \mathrm{HfO}_{2}$.

The origin of the ferroelectricity is attributed to a noncentrosymmetric orthorhombic space group which is only

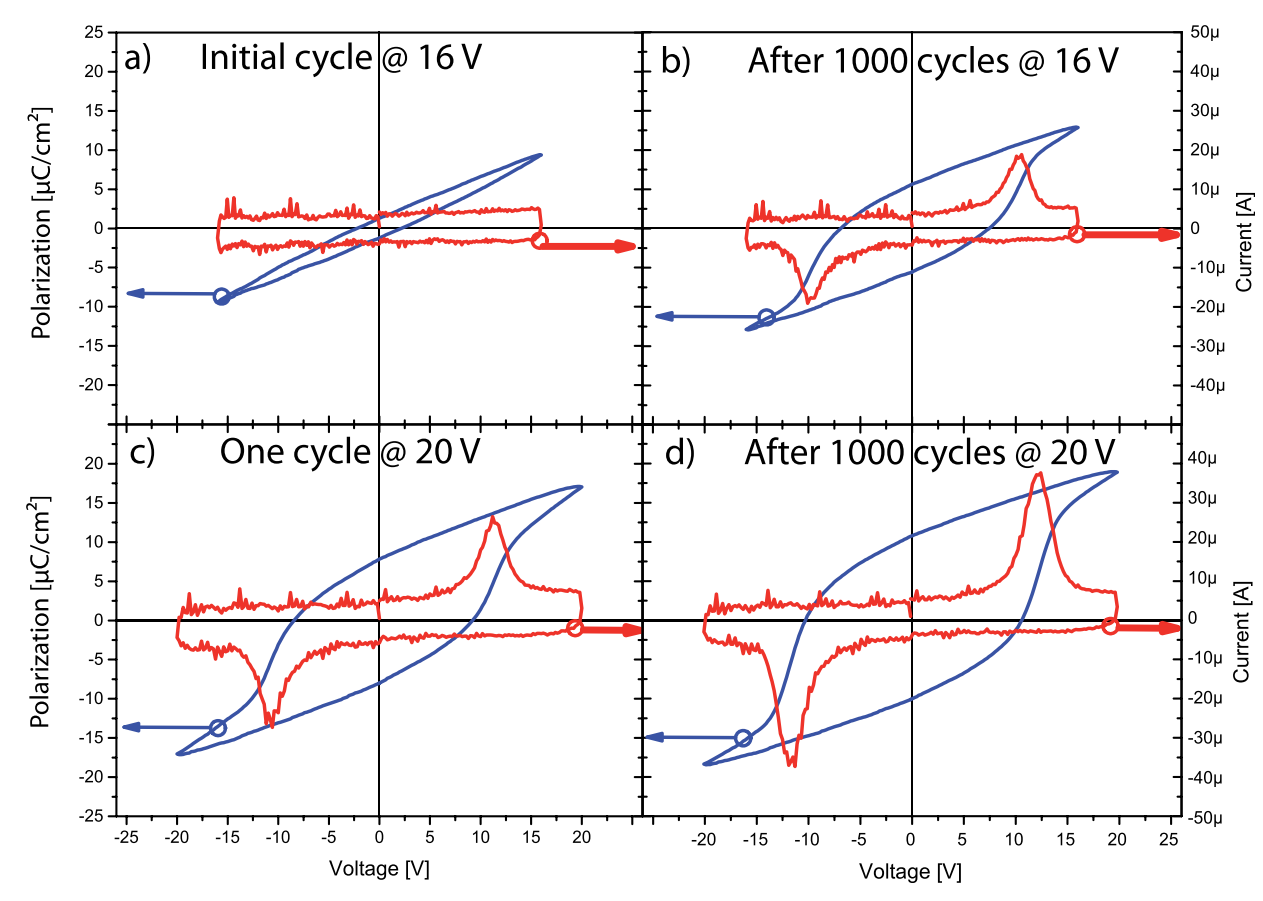

FIG. 3. The influence of "wake up" cycles are exemplarily shown for a $56 \mathrm{~nm}$ thick Y: $\mathrm{HfO}_{2}$ layer. Initially, no ferroelectric switching is observed (a). After 1000 cycles a hysteresis curve appears with distinctive current peaks, indicating the ferroelectric switching (b). Further cycling at higher voltages leads to an improvement of the remanent polarization (c) and (d). 


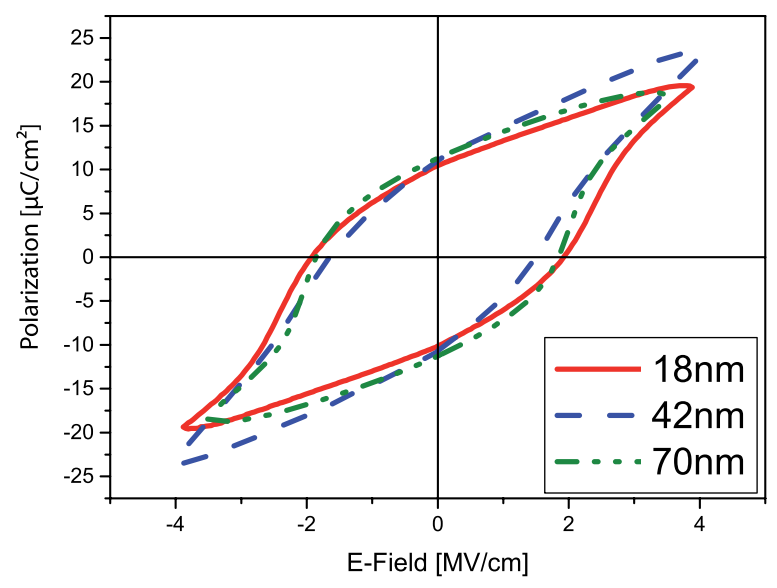

FIG. 4. P-V curves for different thicknesses of the $\mathrm{Y}: \mathrm{HfO}_{2}$ layer. No significant influence of the layer thickness on the polarization is observed at all.

stable at particular conditions. ${ }^{4}$ Fig. 5 shows the grazing incidence XRD diffractograms for two samples with a layer thickness of $18 \mathrm{~nm}$ and $70 \mathrm{~nm}$, respectively. Both diffractograms reveal a clear cubic phase without any hints at an orthorhombic phase. An explanation could be correlated to the wake up cycles needed to obtain a hysteresis. During the cycling a field-driven phase transition might occur, leading to a non-centrosymmetric ferroelectric phase. Field induced phase transition to a ferroelectric phase is reported in Ref. 18. Such a transition was not found in GI-XRD spectra performed on the samples before and after the wake-up cycles. That could be due to the small cross section observed during the measurement.

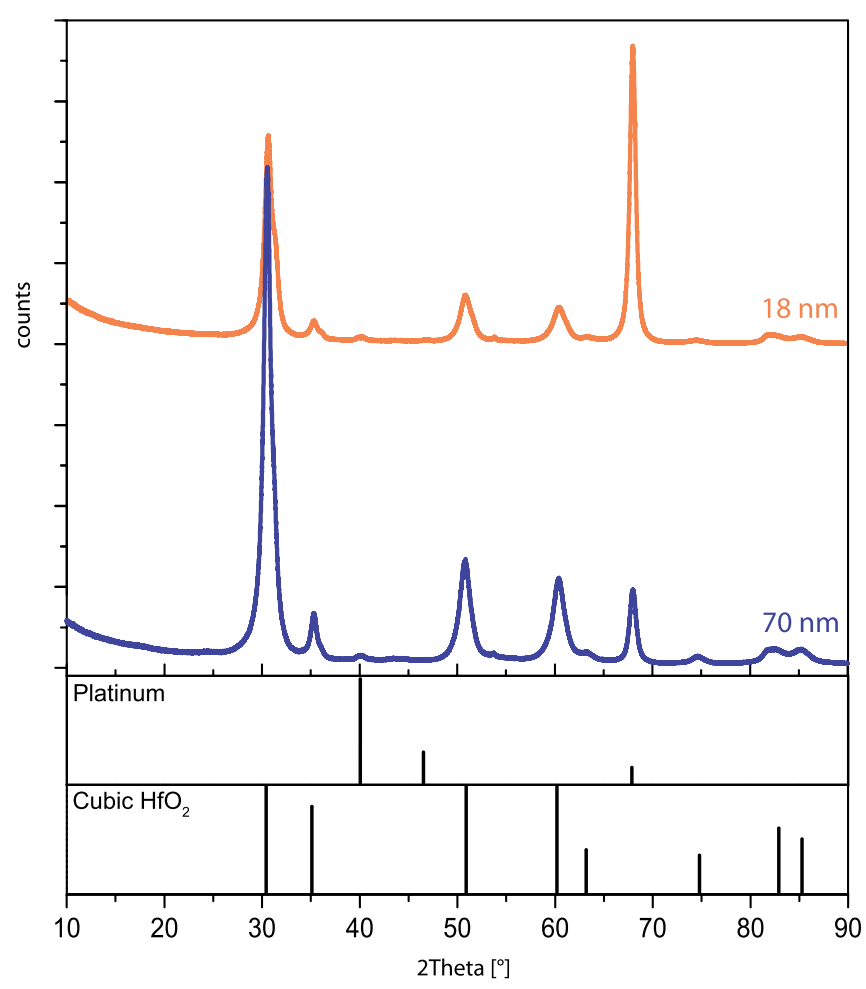

FIG. 5. GI-XRD patterns of $18 \mathrm{~nm}$ and $70 \mathrm{~nm}$ thick $\mathrm{Y}: \mathrm{HfO}_{2}$ on platinum. Both samples show almost the same diffractograms with a definite cubic phase and no evidence measured of a monoclinic or orthorhombic phase. A grain size of approximately $10 \mathrm{~nm}$ was calculated for the $70 \mathrm{~nm}$ thick sample using the Scherrer equation.
In summary, we have fabricated ferroelectric yttrium doped hafnium oxide films with chemical solution deposition using platinum electrodes. Due to the deposition procedure thicker films up to $70 \mathrm{~nm}$ were produced without showing any decrease of the polarization. This was attributed to the step by step annealing after each spinning of the solution resulting in a layered $\mathrm{Y}: \mathrm{HfO}_{2}$ film. The deposition of thicker films has the potential for piezoelectric sensors and actuators based on hafnium oxide. It was shown that wake up cycles do not only improve the remanent polarization but are also essential for the forming of a hysteresis. This necessarily cycling was assumed to correlate with the fact that initially a cubic phase was found. Due to cycling a phase transition could occur to a ferroelectric non-centrosymmetric phase. Further work to prove this hypothesis is in progress.

This work was funded by the German Research Foundation (Deutsche Forschungsgemeinschaft) within the scope of the project "Inferox" (Project No. MI 1247/11-1).

${ }^{1}$ K. Kim and S. Lee, "Integration of lead zirconium titanate thin films for high density ferroelectric random access memory," J. Appl. Phys. 100, 051604 (2006).

${ }^{2}$ M. Qazi, M. Clinton, S. Bartling, and A. P. Chandrakasan, "A low-voltage $1 \mathrm{Mb}$ FRAM in $0.13 \mu \mathrm{m}$ CMOS featuring time-to-digital sensing for expanded operating margin," IEEE J. Solid-State Circuits 47, 141-150 (2012).

${ }^{3}$ Nanoelectronics and Information Technology, 3rd ed., edited by R. Waser (Wiley-VCH, 2012).

${ }^{4}$ T. S. Boescke, J. Mueller, D. Braeuhaus, U. Schroeder, and U. Boettger, "Ferroelectricity in hafnium oxide thin films," Appl. Phys. Lett. 99, 102903 (2011).

${ }^{5}$ J. Mueller, U. Schroeder, T. S. Boescke, I. Mueller, U. Boettger, L. Wilde, J. Sundqvist, M. Lemberger, P. Kuecher, T. Mikolajick, and L. Frey, "Ferroelectricity in yttrium-doped hafnium oxide," J. Appl. Phys. 110, 114113 (2011).

${ }^{6}$ J. Mueller, T. S. Boescke, D. Braeuhaus, U. Schroeder, U. Boettger, J. Sundqvist, P. Kuecher, T. Mikolajick, and L. Frey, "Ferroelectric $\mathrm{Zr}(0.5) \mathrm{Hf}(0.5) \mathrm{O}(2)$ thin films for nonvolatile memory applications," Appl. Phys. Lett. 99, 112901-1-112901-3 (2011).

${ }^{7}$ S. Mueller, J. Mueller, A. Singh, S. Riedel, J. Sundqvist, U. Schroeder, and T. Mikolajick, "Incipient ferroelectricity in Al-doped $\mathrm{HfO} 2$ thin films," Adv. Funct. Mater. 22, 2412-2417 (2012).

${ }^{8}$ S. Mueller, C. Adelmann, A. Singh, S. Van Elshocht, U. Schroeder, and T. Mikolajick, "Ferroelectricity in Gd-doped HfO2 thin films," ECS J. Solid State Sci. Technol. 1, N123-N126 (2012).

${ }^{9}$ T. Schenk, S. Mueller, U. Schroeder, R. Materlik, A. Kersch, M. Popovici, C. Adelmann, S. Van Elshocht, and T. Mikolajick, "Strontium doped hafnium oxide thin films: Wide process window for ferroelectric memories," in ESSDERC, 2013.

${ }^{10}$ T. Olsen, U. Schroeder, S. Mueller, A. Krause, D. Martin, A. Singh, J. Mueller, M. Geidel, and T. Mikolajick, "Co-sputtering yttrium into hafnium oxide thin films to produce ferroelectric properties," Appl. Phys. Lett. 101, 082905-1-082905-4 (2012).

${ }^{11}$ T. Schneller, R. Waser, M. Kosec, and D. Payne, Chemical Solution Deposition of Functional Oxide Thin Films (Springer, 2013).

${ }^{12} \mathrm{~T}$. Schneller and R. Waser, "Chemical solution deposition of ferroelectric thin films-state of the art and recent trends," Ferroelectrics, Switzerland 267, 293-301 (2002).

${ }^{13}$ P. Gerber, A. Roelofs, O. Lohse, C. Kugeler, S. Tiedke, U. Boettger, and R. Waser, "Short-time piezoelectric measurements in ferroelectric thin films using a double-beam laser interferometer," Rev. Sci. Instrum. 74, 2613-2615 (2003).

${ }^{14}$ D. Bolten, O. Lohse, M. Grossmann, and R. Waser, "Reversible and irreversible domain wall contributions to the polarization in ferroelectric thin films," Ferroelectrics, Switzerland 221, 251-257 (1999).

${ }^{15}$ V. V. Afanas'ev, A. Stesmans, L. Pantisano, S. Cimino, C. Adelmann, L. Goux, Y. Y. Chen, J. A. Kittl, D. Wouters, and M. Jurczak, "TiN $\mathrm{T}_{\mathrm{x}} / \mathrm{HfO}_{2}$ interface dipole induced by oxygen scavenging," Appl. Phys. Lett. 98, 132901 (2011). 
${ }^{16}$ D. Zhou, J. Xu, Q. Li, Y. Guan, F. Cao, X. Dong, J. Mueller, T. Schenk, and U. Schroeder, "Wake-up effects in Si-doped hafnium oxide ferroelectric thin films," Appl. Phys. Lett. 103, 192904 (2013).

${ }^{17}$ M. H. Park, H. J. Kim, Y. J. Kim, T. Moon, and C. S. Hwang, "The effects of crystallographic orientation and strain of thin $\mathrm{Hf} 0.5 \mathrm{Zr}$
0.502 film on its ferroelectricity," Appl. Phys. Lett. 104, 072901 (2014).

${ }^{18}$ X. Tan, J. Frederick, C. Ma, W. Jo, and J. Roedel, "Can an electric field induce an antiferroelectric phase out of a ferroelectric phase?" Phys. Rev. Lett. 105, 255702 (2010). 